\title{
A Case of Mucocele Gallbladder with Review of Literature
}

\author{
Dr. Bhavna Valecha*
}

Resident, Department of Pathology, BPS GMC for Women, Ganaur - Gohana Rd, Khanpur Kalan, Haryana 131305, India

\author{
DOI: $10.36348 / \mathrm{sipm} .2019 . \mathrm{v} 04 \mathrm{i11.012}$ \\ | Received: 18.11.2019| Accepted: 25.11.2019| Published: 28.11.2019 \\ *Corresponding author: Dr. Bhavna Valecha
}

\section{Abstract}

Introduction: The term gallbladder mucocele refers to an overdistended gallbladder filled with mucoid or clear and watery content. Usually noninflammatory, it results from outlet obstruction of the gallbladder and is commonly caused by an impacted stone in the neck of the gallbladder or in the cystic duct. Case Report: A Patient 27 yrs female came with pain in abdomen to the opd, the patient presented with 3 day history of vomiting and inappetance. The ultrasound was done and chronic cholecystitis with cholelithiasis diagnosis was given and the patients were operated and the laproscopic cholecystectomy specimens were sent to pathology department. Laparoscopic cholecystectomy was performed and the gall bladder was sent for routine histopathological evaluation. The diagnosis of mucocele was made on histopathological examination.

Keywords: Mucocele Gallbladder, inappetance, Laparoscopic cholecystectomy.

Copyright @ 2019: This is an open-access article distributed under the terms of the Creative Commons Attribution license which permits unrestricted use, distribution, and reproduction in any medium for non-commercial use (NonCommercial, or CC-BY-NC) provided the original author and sources are credited.

\section{INTRODUCTION}

Gallbladder mucocele is the distention of the gallbladder by an inappropriate accumulation of mucus.

Decreased bile flow, decreased gallbladder motility, and altered absorption of water from the gallbladder lumen are predisposing factors to biliary sludge. Biliary sludge may be a precipitating factor for the development of canine biliary mucoceles. However, it is more likely to be a small part of a complex disease process involving inflammation of the gallbladder wall and changes to the lining of the gallbladder changing the consistency of its secretions.

Hypersecretion of mucus leads to an accumulation of thick gelatinous bile within the gall bladder. Increased viscosity over a period of weeks or months leads to thick gelatinous material eventually occupying the entire lumen of the gallbladder and in some cases also being present in the ducts.

Mucocele of the gallbladder is a condition caused by the prolonged blockage of the cystic duct, usually by an impacted gallstone. Another name for this condition is hydrops of the gallbladder. It is most often not identified before surgery but is an incidental finding at the time of either laparoscopic or open cholecystectomy. This diagnosis is made when the gallbladder is surgically decompressed, and clear mucous-like fluid has replaced the green or brown bile. Patients present with signs and symptoms of acute or chronic cholecystitis.

\section{CASE REPORT}

Patient 27 yrs female came with pain in abdomen to the opd, the patient presented with 3 day history of vomiting and inappetance.

Physical examination revealed 5-7\% dehydration and icteric sclera. Abdominal palpation was unremarkable and he was not overtly painful on palpation.

CBC showed mild anemia (HCT 32\%) and neutrophilia (neutrophils 24,000). Chemistry profile showed marked elevation of liver enzymes (ALP 4136 U/L, ALT 935 U/L, GGT 54 U/L) and elevation of total bilirubin $(4.7 \mathrm{mg} / \mathrm{dl})$. A complete diagnostic abdominal ultrasound was requested and the following images were obtained. The ultrasound was done and chronic cholecystitis with cholelithiasis diagnosis was given and the patients were operated and the cholecystectomy specimens were sent to pathology department. Laparoscopic cholecystectomy was performed and the gall bladder was sent for routine histopathological evaluation. The diagnosis of mucocele was made on histopathological examination. 


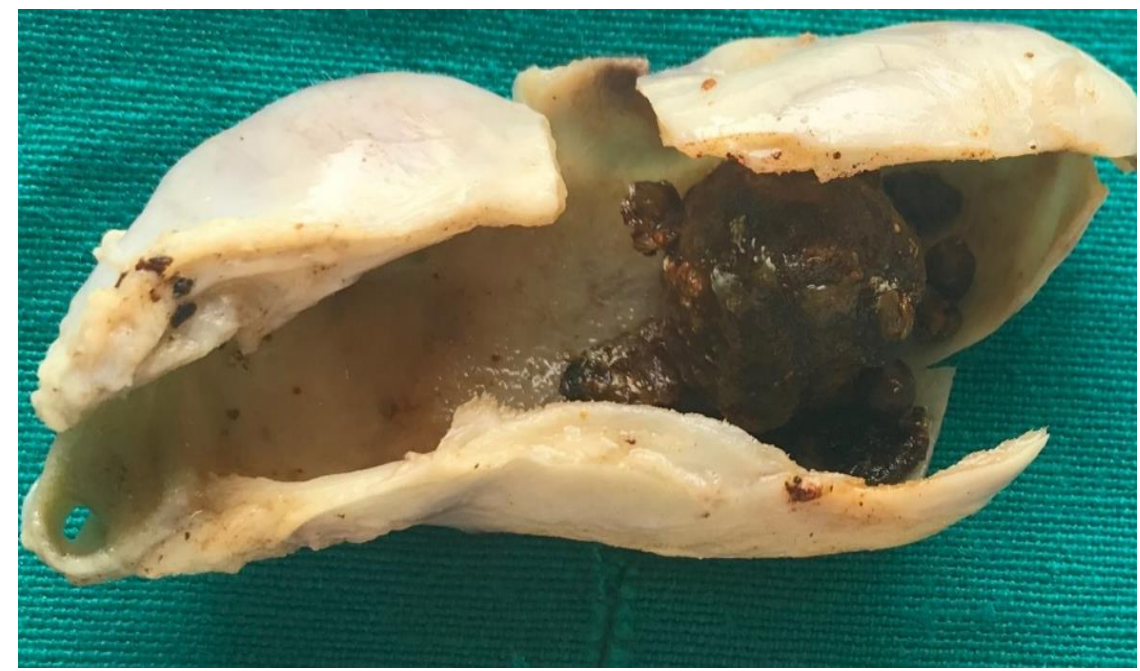

Showing Mucocele of Gall Bladder and Thinning of the Wall

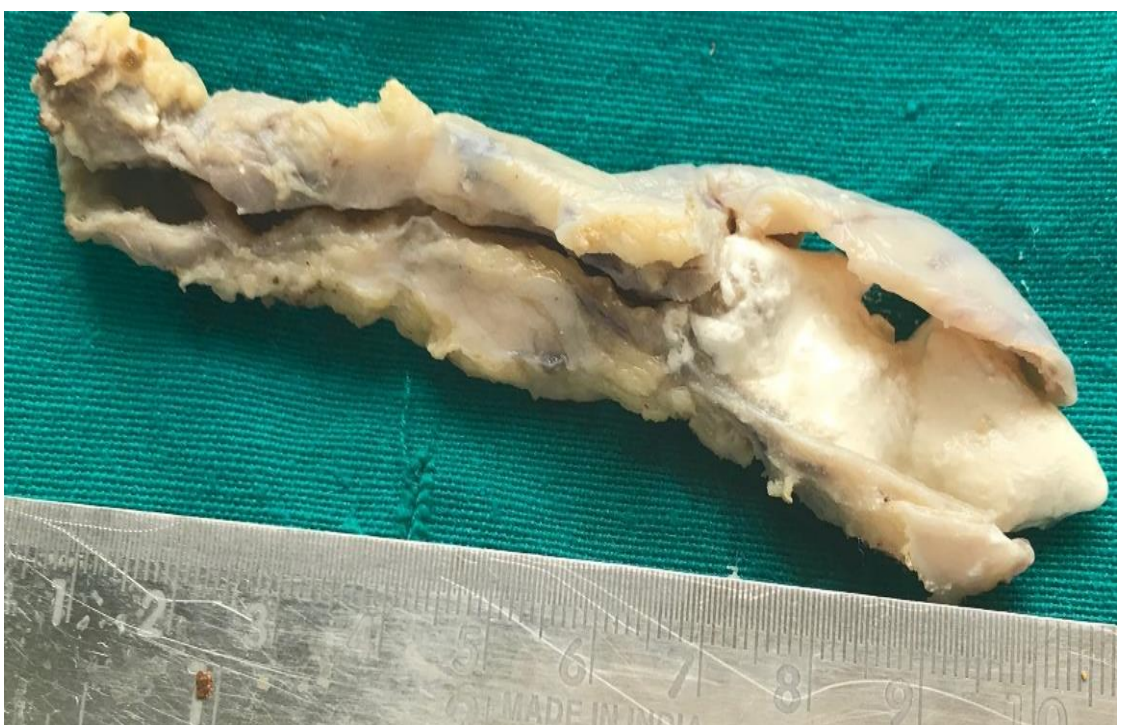

Gross Showing Mucocele of Gall Bladder and Thinning of the Wall

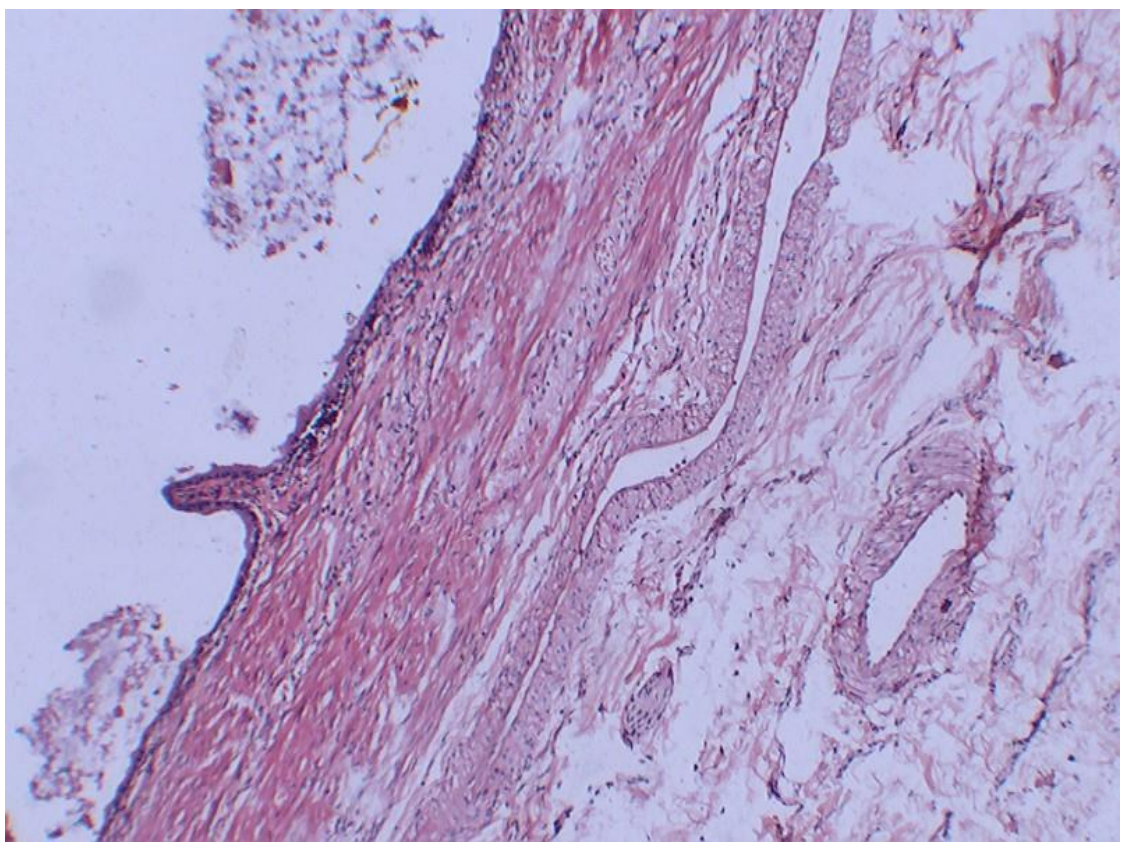

Photomicrograph Showing Mucocele Showing Flattened Epithelium 40X, H\&E 


\section{Gross Features}

The gall baldder were elongated to $8.5 \mathrm{~cm}$. After cutting open whitish material came out. The lumen contained mixed stones. The wall thickness was decreased and was measured $1.5 \mathrm{~cm}$.

\section{Microscopy}

- Fibrous replacement of muscular wall

- Thin wall with flattened epithelium and sparse inflammation

\section{DISCUSSION \\ EPIDEMIOLOGY}

Mucoceles can occur in any patient with acute calculous or acalculous cholecystitis. Gallbladder disease occurs in men and women. Certain populations are more prone to gallbladder disease. The risk of gallbladder disease increases in women, obese patients, pregnant women, and patients in their 40s. Drastic weight loss or acute illnesses may also increase the risk, as well as a family propensity for this condition and the formation of gallstones. Other conditions that cause breakdown of blood cells, for example, Sickle Cell disease, also increase the incidence of gallstones Gallstones also have a hormonal association. Estrogen has been shown to increase bile cholesterol as well as decrease gallbladder contractility. Women of reproductive age or on birth control medication containing estrogen have a two-fold increase in gallstone formation compared to men. People with chronic illness such as diabetes also have an increase in gallstone formation as well as reduced gallbladder wall contractility due to neuropathy [2].

\section{ETIOLOGY}

A malfunctioning gallbladder causes cholecystitis. The liver makes bile which travels down the bile duct, to be stored in the gallbladder. After eating certain foods, especially spicy or greasy foods, the gallbladder is stimulated to empty the bile out of the gallbladder, through the cystic duct, down the bile duct into the duodenum. This process aids in food digestion. If the gallbladder is improperly functioning, the bile may not empty completely which may lead to stone formation. Gallstones can cause mechanical blockage of the cystic duct. The gallbladder also serves to concentrate bile and reabsorb water from the bile. The gallbladder can store up to $1500 \mathrm{~mL}$ of bile and can distend if there is an outlet obstruction. If this outlet obstruction is complete, usually from an impacted gallstone in the cystic duct, the bile salts will get reabsorbed by the gallbladder mucosa over time and be replaced by clear, watery mucus. Other etiologies of gallbladder mucocele include anything that causes a blockage of the cystic duct or distal biliary tree. Neoplasms including gallbladder polyps and tumors can result in hydrops. Congenital strictures, gallbladder parasites, and external compression from liver disease or tumors can also cause gallbladder mucoceles. Other factors that can lead to hydrops include anything that increases the risk of cholecystitis such as drastic weight reduction, prolonged TPN, gastric surgeries with disruption of the vegas nerves, critical illness, diabetes, hyperlipidemia, hypercalcemia, and biliary conditions such as Carole disease. Patients with acute acalculous cholecystitis can also form gallbladder mucoceles [1].

\section{HISTORY}

Cases of chronic cholecystitis present with progressing right upper abdominal pain with bloating, food intolerances (especially greasy and spicy foods), increased gas, nausea, and vomiting. Pain in the midback or shoulder may also occur. Patients usually present with a history including several days of progressive symptoms [4].

\section{EVALUATION}

A physical exam with a comprehensive history is paramount in making the diagnosis of cholecystitis. A complete blood count and a comprehensive metabolic panel are also important. In cases of chronic cholecystitis, these results may be within the reference range. In acute cholecystitis or severe disease, white blood cell count may be elevated. Liver enzymes may also be elevated. If the bilirubin level is above 2, then consider a possible common bile duct stone. Even in the presence of severe gallbladder disease, lab values may be within the reference range. Amylase and lipase must also be checked to rule out pancreatitis [5].

\section{HISTOPATHOLOGY}

Most often, an impacted gallstone is located in the cystic duct or Hartmann pouch. This stone is usually singular, but multiple stones can also be present. Cases caused by other etiologies such as polyps, tumors, or parasites exhibit these findings in pathology. Extreme or prolonged cases can lead to actual perforation of the gallbladder [3].

\section{DIFFERENTIAL DIAGNOSIS}

Many other conditions can mimic gallbladder disease. Patients who present with acute biliary colic are often worked up for cardiac issues. Other common conditions with similar presenting symptoms are peptic ulcer disease, irritable bowel disease, inflammatory bowel disease, gastroesophageal reflux disease, pulmonary embolism, and musculoskeletal disorders. The diagnosis of acute cholecystitis with possible hydrops in patients with a palpable gallbladder, Murphy sign, and a positive gallbladder ultrasound should not be confused with any other diagnosis [6].

\section{REFERENCES}

1. Chaly, T., Campsen, J., O'Hara, R., Hardman, R., Gallegos-Orozco, J. F., Thiesset, H., \& Kim, R. D. (2017). Mucocele mimicking a gallbladder in a transplanted liver: A case report and review of the literature. World journal of transplantation, 7(6), 359-363. 
2. Shirah, B. H., Shirah, H. A., \& Albeladi, K. B. (2018). The value of intraoperative percutaneous aspiration of the mucocele of the gallbladder for safe laparoscopic management. Updates in surgery, 70(4), 495-502.

3. Jansen, C., Halliburton, P., Dibble, S., \& Dodd, M. J. (1993). Family problems during cancer chemotherapy. In Oncol Nurs Forum, 20(4):68994.

4. Clemente, G., Fico, V., De Sio, D., \& De Rose, A M. (2017). The Mucocele of the Gallbladder. Journal Gastrointest Surgery. 21(8):1366-1367.
5. Limaiem, F., Sassi, A., Talbi, G., Bouraoui, S., \& Mzabi, S. (2017). Routine histopathological study of cholecystectomy specimens. Useful? A retrospective study of 1960 cases. Acta Gastroenterol. Belg, 365-370.

6. Reyes, Q., McLeod, R. L., Fernandes, K., Muralidharan, V., \& Weinberg, L. (2017). Magnetic resonance cholangiopancreatography uncovering massive gallbladder mucocele in a patient with ambiguous clinical and laboratory findings: A case report. International journal of surgery case reports, 36, 133-135. 